Check for updates

Cite this: RSC Adv., 2017, 7, 23238

Received 21st February 2017 Accepted 6th April 2017

DOI: 10.1039/c7ra02138k

rsc.li/rsc-advances

\section{Effects of naturally occurring flavonoids on ferroportin expression in the spleen in iron deficiency anemia in vivo}

\author{
Maryam Mazhar, ${ }^{\text {a }}$ Shaheen Faizi, ${ }^{\text {b }}$ Anum Gul, ${ }^{a}$ Nurul Kabir ${ }^{a}$ \\ and Shabana U. Simjee (iD *ab
}

\begin{abstract}
Anemia due to iron deficiency is one of the unsolved issues around the globe for which oral iron supplementation is the common treatment. However, the consumption of complex diets plays an important role in modulating iron homeostasis. In this study, we aimed to examine the interaction of ubiquitous polyphenol, quercetin and related flavonoids with iron supplements in an animal model of iron deficiency anemia (IDA). Female weanling Sprague Dawley rats were kept on a low iron diet for 20 days to induce iron deficiency anemia followed with $50 \mathrm{mg} \mathrm{kg}^{-1}$ ferrous sulfate (FS) supplement combined in equal ratio with quercetin (Que), quercetagetin (Qtg), and patuletin (Pat) for 30 days. Besides evaluation of basic hematological parameters, we mainly investigated changes in expression of splenic ferroportin by immunohistochemistry. Our data indicate that each polyphenol shows different outcomes and regulates iron variably which might result from differences in their structures. However, the combination of various flavonoids and iron significantly increases hemoglobin, serum iron, splenic iron stores and ferroportin as compared to a model group. The QtgFS combination was found to show more control over blood parameters. Splenic iron stores achieved with the QueFS and QtgFS combinations were found to increase similarly to with FS alone. Ferroportin expression was increased as in the order QueFS < QtgFS < PatFS where quercetin showed comparatively strict control over ferroportin regulation when administered with FS compared to the other flavonoid iron combinations. This variation in regulation needs to be explained further. To conclude, different polyphenols regulate ferroportin expression and ultimately iron homeostasis differently and should be further evaluated for their effect on iron regulation.
\end{abstract}

\section{Introduction}

A lack of iron in the diet is one of the leading causes of iron deficiency anemia in developing countries. The source of food consumed by populations in low income countries is usually based on plants and cereals. ${ }^{1}$ Flavonoids are one of the biggest classes of secondary metabolites produced by plants where quercetin is the ubiquitous flavonol widely distributed in fruits and vegetables consumed on daily basis.,3 Moreover consumption of a plant based diet or cereals may also render the population with gastrointestinal alterations such as changes in gut microbiota, celiac disease and ultimately malabsorption of nutrients including iron, folic acid, calcium and fat-soluble

${ }^{a}$ Dr Panjwani Center for Molecular Medicine and Drug Research, International Center for Chemical and Biological Sciences, University of Karachi, Karachi-75270, Pakistan ${ }^{b}$ H.E.J. Research Institute for Chemistry, International Center for Chemical and Biological Sciences, University of Karachi, Karachi-75270, Pakistan. E-mail: shabana.simjee@iccs.edu; sho1us@hotmail.com; Tel: +92 21 99261701; +92 21 99261702; +92 21 34824930; +92 21 34824934; +92 21 34824936; +92 21 4824901; $+92214824902$ vitamins. $^{4,5}$ Conventional supplement therapy with iron salts is the common, effective and economic method of choice for the treatment of iron deficiency anemia. ${ }^{6}$

The micronutrient iron is required in trace amounts for performing various functions in the body. ${ }^{7}$ It is known for its redox functions and very narrow limit of regulation. ${ }^{8}$ An orchestra of proteins at different levels is dedicated to bring about a tight control over iron balance and carry out import inside a cell, export out of cell, transport through circulation and binding for storage. ${ }^{9}$ An iron exporter, ferroportin, is a transmembrane protein present on the membrane surfaces of different cells in duodenum, spleen, liver, macrophages and placenta. $^{10}$ Iron overload or iron deficiency modulate the expression of ferroportin regulated at different levels of protein and gene expression. ${ }^{11}$ Depending on specific iron related function of each organ; hepatocytes, intestinal enterocytes, macrophages and erythroid cells, ferroportin expression is varied. For example FPN1B is increased in duodenum in iron deficiency anemia in order to increase iron efflux and transport to the body but very little is known about splenic ferroportin expression or in other organs. ${ }^{12}$ Though, there are some studies 
which evidenced that ferroportin expression is translationally repressed in iron deficiency anemia in cells. ${ }^{\mathbf{1 2 , 1 3}}$ Moreover, there is a lack of in vivo based results and mostly in vitro studies have been conducted to evaluate ferroportin expression in iron deficiency anemia.

Quercetin is one of the most abundantly consumed dietary components present in our complex food..$^{\mathbf{1 4}}$ Nowadays, quercetin is used as a nutraceutical because of its antioxidant properties. ${ }^{15} \mathrm{In}$ cases where the anemic individual is having iron treatment and consuming food containing flavonoids, we need to know how quercetin and structural analogues of quercetin i.e., patuletin and quercetagetin having $\mathrm{OH}$ at position 3 and 6, interact with iron stores and ferroportin expression (Fig. 1). Iron complex with casein $^{16}$ and lactoglobulin ${ }^{17}$ has been found to promote iron absorption and release in controlled manner as well as decreased side effects as compared to ferrous sulfate therapy alone for the treatment of iron deficiency anemia. ${ }^{18}$ Iron complexes with flavonoids ${ }^{19}$ are known to inhibit intestinal iron absorption and decrease ferroportin expression ${ }^{20}$ though some studies report vice versa. ${ }^{21}$ Recently, quercetin has been found to act as a vehicle for iron transport in and out of cell. Complexation of iron with flavonoids may prove beneficial in preventing iron induced side effects such as Fenton reaction, mucosal block and gastrointestinal problems. ${ }^{22}$

Polyphenols are widely distributed naturally in food and its interaction with iron is controversial. In this study, we were interested to know that in case of iron deficiency anemia when iron regulatory system is stimulated to restore iron, how the polyphenols that we consume as a part of our complex diet interfere with the effect of iron supplementation and exert their effects on key players of iron homeostasis. In order to mimic the nutritional iron deficiency anemia, rats were weaned on low iron diet initially for twenty days. Subsequently, quercetin, quercetagetin and patuletin polyphenols were administered orally with or without iron supplementation in equal ratio. Blood parameters and splenic SLC40 expression were mainly investigated and we found that ferroportin expression is variably regulated by each polyphenol that may depend on their structure or direct influence on ferroportin gene expression. Therefore, polyphenols should be further evaluated for their effect on iron regulation and key players of iron homeostasis.

\section{Methods}

\subsection{Chemicals and reagents}

Low iron diet (Research Diets Inc. NJ, USA), quercetin (Sigma), Giemsa stain (Carl RothGmBH, Germany); Perl's iron stain (Sigma, MO, USA), primary rabbit polyclonal antibody antiSLC40A1 (abcam®, Cambridge, MA), secondary antibody Texas Red conjugated goat anti-rabbit 594 IgG (Jackson ImmunoResearch Laboratories Inc., PA, USA), DAPI (Wako Pure Chemical Industries, Ltd., Japan), Roti-immunoblock and Mowiol (Carl RothGmBH, Germany).

\subsection{Plant materials}

Quercetagetin and patuletin were isolated from freshly collected, shade dried flowers of Tagetes erecta and Tagetes patula respectively. An authenticated voucher specimen number KUH GH no. 67280 for Tagetes patula and KUH GH 67281 for Tagetes erecta was submitted in the herbarium of Department of Botany, University of Karachi.

\subsection{Experimental design}

All experimental procedures were performed in accordance with the NIH guidelines for the care and use of laboratory animals (NIH publication no. 85-23 Rev. 1985) and it was further approved by the Institutional Animal Care and Use Committee of International Center for Chemical and Biological Sciences (ICCBS), University of Karachi (Karachi, Pakistan). Animals had a free access to standard laboratory chow and drinking tap water, housed under controlled laboratory conditions at $22{ }^{\circ} \mathrm{C}$ to $26{ }^{\circ} \mathrm{C}$ and 12 hour light/dark cycles maintained in the pathogen free environment in the animal house facility of ICCBS, University of Karachi.

Female Sprague Dawley rats at post-weaning stage were used for the present study. Iron deficiency anemia was induced by feeding low iron diet ( $\mathrm{Fe}=5 \mathrm{ppm})$ ad libitum for 20 days. Anemic rats were divided into eight groups: group I received no treatment; group II received Que, group III Qtg; group IV Pat; group $\mathrm{V} \mathrm{FeSO}_{4}$; group VII Que/FeSO ${ }_{4}$; group VII Qtg/FeSO group VIII Pat $/ \mathrm{FeSO}_{4}$. Along with consumption of low iron diet throughout the study period, each treatment group received a dose of $50 \mathrm{mg}$ per $\mathrm{kg}$ per body weight by oral gavage continued for a month. The rats were monitored throughout the study for<smiles>O=c1c(O)c(-c2ccc(O)c(O)c2)oc2cc(O)cc(O)c12</smiles>

\begin{tabular}{|l|c|}
\hline \multicolumn{1}{|c|}{ Compounds } & $\mathrm{C}_{6}$ Position \\
\hline Quercetin & $\mathrm{H}$ \\
\hline Quercetagetin & $\mathrm{OH}$ \\
\hline Patuletin & $\mathrm{OCH}_{3}$ \\
\hline
\end{tabular}

Fig. 1 Structure of the flavonoids evaluated in this study. 
changes in external signs of anemia and hemoglobin level at regular interval. Control animals received free access to normal laboratory rodent diet containing $350 \mathrm{ppm}$ iron. Anemic, treated and age-matched normal rats were sacrificed after completion of the one month therapy. Blood was collected through cardiac puncture for hematological tests. Spleen was immediately removed and fixed for histological studies.

\subsection{Peripheral blood smear}

The peripheral blood films were made manually by collecting blood from tail nick on a clean slide, fixed with methanol and stained with Wright-Giemsa observed under bright field microscope oil immersion lens $\times 100$.

\subsection{Hematological analysis}

Hemoglobin level was measured on every 5 th day and at the end of the whole experiment using portable digital hemoglobinometer (Mission, CA). Blood for serum iron estimation was obtained by cardiac puncture at the time of animal sacrifice collected in plain glass tubes, centrifuged at $4000 \mathrm{rpm}$ for 30 minutes in order to separate serum stored in separate labeled tubes at $-20{ }^{\circ} \mathrm{C}$. Serum iron was also estimated at the end of experiment by conventional colorimetric assay using Ferrimat kit (bioMérieux, France) as per the manufacturer's instructions.

\subsection{Spleen iron staining}

For histopathological analysis, the isolated spleen was fixed in $10 \%$ neutral buffered formalin and dehydrated in ascending series of graded isopropyl alcohol. Paraffin embedded spleen tissues were cut into $6 \mu \mathrm{m}$ thin sections and stained with Perl' iron stain. The stained sections were examined at $10 \times$ magnification to investigate the changes in iron stores of spleen in response to various treatments used in this study.

\subsection{Immunohistochemistry of ferroportin}

Immunolocalization of ferroportin was performed on the spleen tissue sections. The tissue sections were deparaffinized, hydrated, and perfused with PBS for $15 \mathrm{~min}$. The tissue sections were placed in a blocking reagent (Roti-immunoblock) for $25 \mathrm{~min}$ at room temperature to reduce nonspecific staining. Then sections were incubated with anti-SLC40 antibody at $42{ }^{\circ} \mathrm{C}$ for $1 \mathrm{~h}$. After being washed 3 times for $5 \mathrm{~min}$ in PBS, the sections were further incubated with Texas Red conjugated goat antirabbit 594 IgG for $1 \mathrm{~h}, 42{ }^{\circ} \mathrm{C}$. Following washing with PBS 3 times for $5 \mathrm{~min}$, the sections were stained with DAPI for $1 \mathrm{~min}$. Finally, after a wash in PBS, the sections were mounted in Mowiol and kept in dark area.

\subsection{Microscopy and image analysis}

Specimens were observed under Nikon 90i microscope and photomicrographs were captured using a Nikon DXM 1200C camera with NIS-Elements image analysis software AR 3.0 (Nikon, Japan). Adobe Photoshop software was used for image processing.

\subsection{Statistical analysis}

Statistical comparisons were made using the one way analysis of variance (ANOVA) with post hoc analysis using Bonferroni's post hoc test by IBM SPSS Statistics 20 software. All data are expressed as mean \pm standard error mean (SEM), $n=6$. Values of $* p \leq 0.001$ were accepted as statistically significant.

\section{Results}

\subsection{Hematological tests}

Table 1 shows the hemoglobin, hematocrit and serum iron concentration from normal, model control and treated animals. Blood biochemistry showed significant decrease in IDA model as compared to normal control. Whereas groups treated with $\mathrm{FeSO}_{4}$ alone and $\mathrm{FeSO}_{4}$ combined with polyphenols showed significant increase in hematological parameters evaluated.

\subsection{Evaluation of peripheral blood smears}

Normal control group show normochromic and normocytic red blood cells (Fig. 2a). Anemic control group showed hypochromic cells with anisocytosis and poikliocytosis (Fig. 2b). Therapy with iron supplement recovered red blood cells from anemic morphology (Fig. 2c). Treatment with quercetin, quercetagetin and patuletin alone showed no improvement and RBCs appear hypochromic similar to that in model group (Fig. 2d-f respectively). Standard $\mathrm{FeSO}_{4}$ and combination treatment (QueFS, QtgFS, PatFS) normalized the RBCs size and shape with better restoration of hemoglobin than model group (Fig. $2 \mathrm{~g}$-i respectively).

\subsection{Effect of flavonoids on splenic iron storage}

In normal control group, iron is distributed in the red pulp area of spleen tissue that can be seen as blue deposits in Fig. 3a. Iron deficiency anemia group shows depleted iron stores in Fig. 3b. Supplement $\mathrm{FeSO}_{4}$ recovered splenic iron higher than control group Fig. 3c. Quercetin, quercetagetin and patuletin given as a single agent therapy did not increase the iron stores and it was similar to model group (Fig. 3d-f respectively). Combinations of

Table 1 Hemoglobin, hematocrit and serum iron levels. Values are presented as mean \pm S.D $(n=12)$, significantly different from control group $(* p<0.001)$, from IDA model group $(* * p<0.001)$

\begin{tabular}{lclc}
\hline Groups & $\begin{array}{l}\text { Hemoglobin } \\
\mathrm{mg} \mathrm{dl}\end{array}$ & $\begin{array}{l}\text { Hematocrit } \\
\%\end{array}$ & $\begin{array}{l}\text { Serum iron } \\
\mu \mathrm{g} \mathrm{dl}\end{array}$ \\
\hline $\begin{array}{l}\text { Normal } \\
\text { control }\end{array}$ & $14.65 \pm 0.4$ & 43 & $192.4 \pm 7.3$ \\
IDA model & $8.65 \pm 0.5^{*}$ & 25 & $67.2 \pm 7.8^{*}$ \\
Que & $10.19 \pm 0.24$ & 30 & $73.5 \pm 5.9$ \\
Qtg & $10.03 \pm 0.4$ & 29 & $65.9 \pm 4.7$ \\
Pat & $10.15 \pm 0.34$ & 30 & $75.3 \pm 3.6$ \\
FeSO & $15.8 \pm 0.3^{*}$ & 46 & $229 \pm 7.3^{*}$ \\
Que/FeSO $_{4}$ & $15.1 \pm 0.3^{* *}$ & 44 & $201.9 \pm 0.3^{* *}$ \\
Qtg/FeSO $_{4}$ & $14.7 \pm 0.3^{* *}$ & 43 & $181.3 \pm 7.6^{* *}$ \\
Pat/FeSO & $16.1 \pm 0.3^{* *}$ & 47 & $215.4 \pm 7.3^{* *}$
\end{tabular}



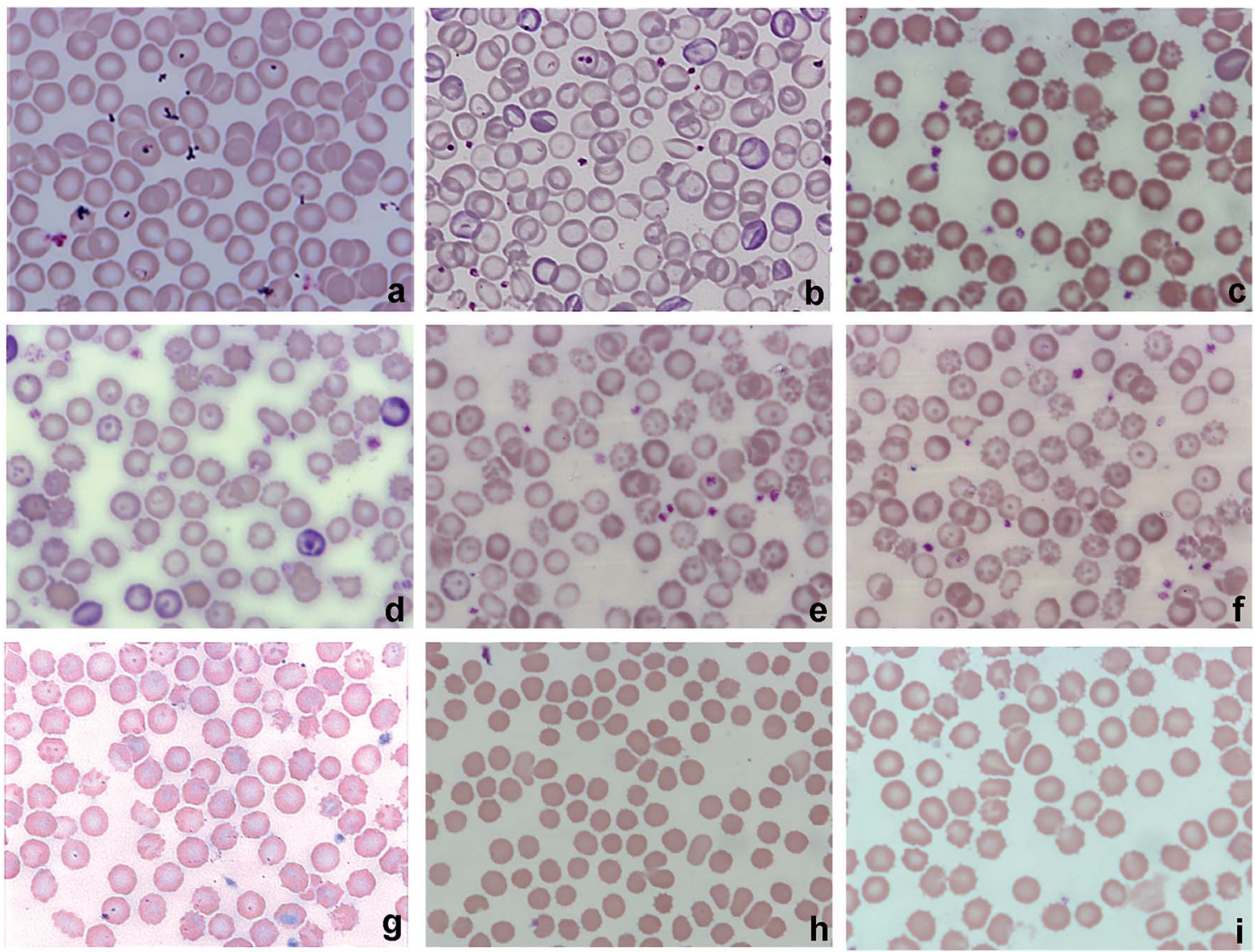

Fig. 2 Peripheral blood smears. (a) Normal control. (b) IDA control. (c) IDA rats treated with FS. (d) IDA rats treated with Que. (e) IDA rats treated with Qtg. (f) IDA rats treated with Pat. (g) IDA rats treated with QueFS. (h) IDA rats treated with $Q$ tgFS. (i) IDA rats treated with PatFS. Scale bar $5 \mu$ m.

quercetin (Fig. 3g) and quercetagetin with iron (Fig. 3h) showed more or less similar iron staining as that achieved by $\mathrm{FeSO}_{4}$ alone. However, the third flavonoid, patuletin, showed relatively dense iron staining than the standard group (Fig. 3i).

\subsection{SLC40 immunohistochemistry}

Normal control spleen shows SLC40 positive immunohistochemistry in red pulp (Fig. 4a). A significant reduction was observed in iron deficient model group (Fig. 4b). Standard treatment increased SLC40 expression as shown in Fig. 4c. Flavonoids given as single agent therapy demonstrated lower expression of SLC40 compared to anemic control group indicating inhibitory effect on SLC40 expression (Fig. 4d-f). However, when administered in combination with $\mathrm{FeSO}_{4}$ an increase was observed with QueFS, QtgFS and PatFS in expression of SLC40 as compared to IDA group (Fig. 4g-i respectively). The quantification data of SLC40 in spleen is shown in Fig. 5.

\section{Discussion}

Iron homeostasis is achieved by a group of proteins working in concert with each other among which ferroportin carry out iron export. Previously conducted in vitro studies have shown that flavonoids make complexes with metal ions thereby inhibiting their absorption through intestine and also have negative regulation on ferroportin..$^{20,23}$ Although flavonoids are widely used for their diverse functions against various ailments, flavonoids present in diet may interact positively or negatively when taken together with drugs or mineral supplement by an individual. Therefore, it is important to determine the effective range of oral intake and pathophysiological condition in which these natural occurring flavonoids are consumed. ${ }^{21}$ In the present study, serum and splenic tissue levels of iron after simultaneous administration of pharmacological doses of iron combined with equal doses of flavonoids were evaluated. Our finding clearly showed that serum and tissues availability of iron increased significantly compared with control group. Therefore, it is assumed that iron sulfate given for the treatment of iron deficiency anemia in the presence of quercetin, quercetagetin and patuletin continue to manage iron absorption.

Quercetin has diverse biological activities including antiinflammatory, antiproliferative, antimicrobial and antiviral properties. High concentrations of quercetin with different conjugated forms and bioavailability are found in apples, green and black teas, onions, kale and red wine. Hollman and coworkers found that the quercetin- $\beta$-glucosides get absorbed far greater than the quercetin without its sugar moiety. The glucosides of quercetin present in onions can absorb more freely than those present in tea and apples. ${ }^{24,25}$ There are few in 

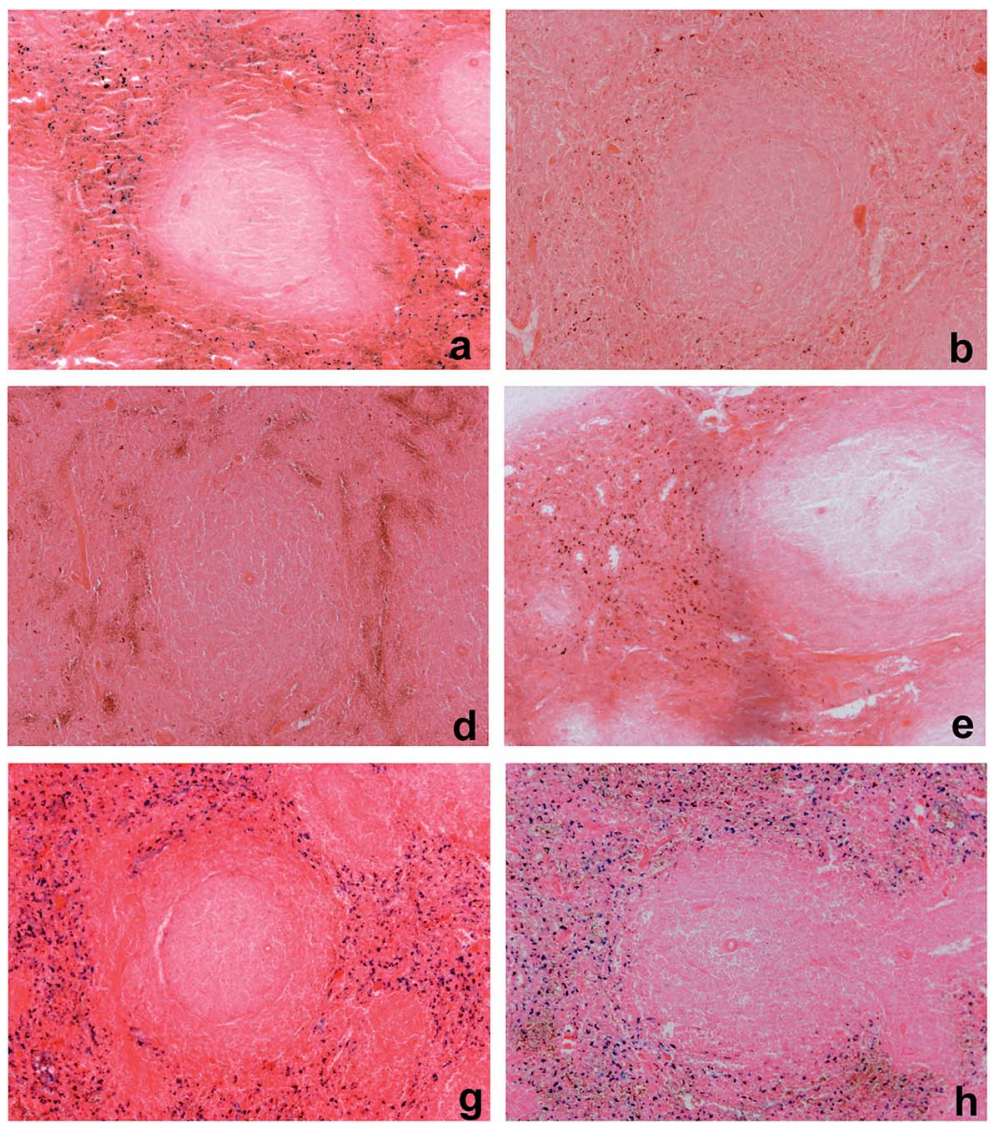
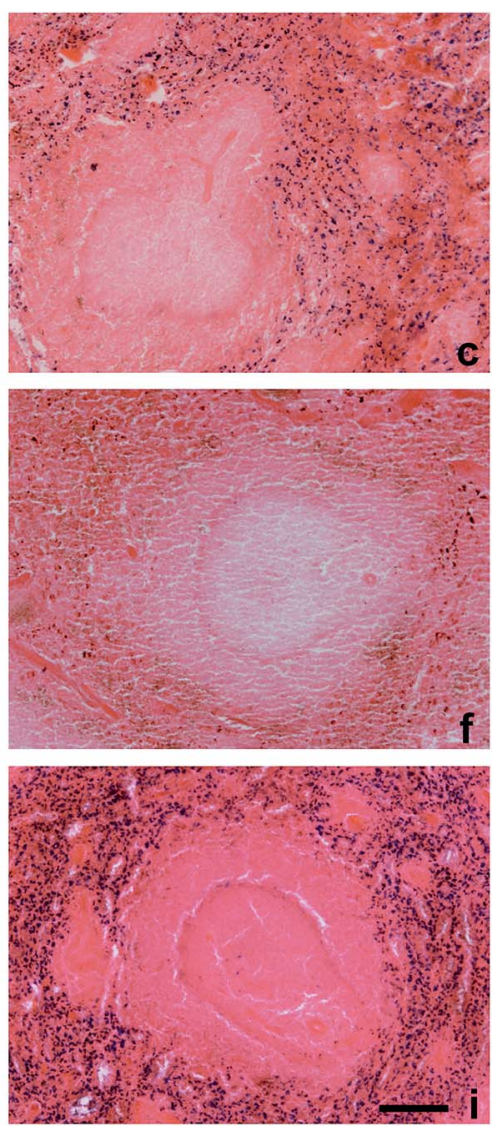

Fig. 3 Effect of flavonoids treatment on iron restoration in iron deficient rats spleen. (a) Normal control. (b) IDA control. (c) IDA rats treated with FS. (d-f) IDA rats treated with Que, Qtg, Pat. (g-i) IDA rats treated with combination of QueFS, QtgFS, PatFS. Scale bar $50 \mu m$.

vivo studies that showed unaltered absorption of divalent metal ions such as zinc and copper in response to acute or prolonged exposure to polyphenols. ${ }^{26}$ In the present study, orally administered doses of combination of flavonoids and $\mathrm{FeSO}_{4}$ (QueFS, QtgFS and PatFS) to rats for the duration of 30 days, improved both serum levels and spleen tissue availability of iron significantly compared to IDA model animals. The underlying mechanism of interaction of polyphenols with iron regulation is a difficult domain to be explained and therefore have resulted in controversial reports. Flavonoids such as rutin, quercetin, kaempferol and morin are known to protect mammalian cellular membranes and structures against oxidative damage mediated by free radicals. ${ }^{27,28}$ In other animal studies, oral ingestion of bioflavonoid antioxidants was found to be effective to protect RBCs from oxidative stresses and resulting damage. ${ }^{29}$ Similar explanation can describe the protective role of the pure flavonoid compounds, quercetin, quercetagetin and patuletin with iron supplementation in iron deficiency anemia used in our study for their protective effect on erythrocyte morphology. The increase in serum and splenic iron stores can be explained based on the fact that formation of metal ion chelate or complex with organic ligands improved their transport across biological membranes and absorption. The positive metallic ion charge is shielded during chelation preventing the interaction of metal ion with negatively charged layer of mucin thereby increasing the lipophilicity and ease to get absorbed from intestinal enterocyte. ${ }^{21}$ Complex of iron with quercetin may provide alternative pathway for iron absorption through glucose transporter. ${ }^{30}$ The reported variation in levels of iron stores and ferroportin in the present study could be related to the differences between structural properties of different flavonoids, including the position and number of hydroxyl groups. This could have a profound effect on the lipophilicity and other physicochemical properties of these flavonoids and also their interaction with trace elements which consequently affect metal homeostasis in a structure-specific fashion. It is possible that flavonoid structure could affect fluid properties of biological membranes that could affect permeability and cell membrane associated enzymatic activities. $^{31}$ Ferroportin is a multi-spanning membrane protein which shows tissue-specific differences in gene regulation thus further studies required..$^{32}$ Iron responsive element at untranslated $5^{\prime}$ end of ferroportin ensures translational repression through iron regulatory proteins in iron deprived cellular states. ${ }^{12}$ Consistent with a recent study, iron deficiency adapt cellular machinery for a compensatory mechanism to increase iron accessibility by decreasing the translation of ferritin and ferroportin mRNAs. On the other hand, large iron pools lead to increased translation of ferritin and ferroportin to enhance the release of iron. ${ }^{33}$ Other studies have also reported increased ferroportin in response to iron at 

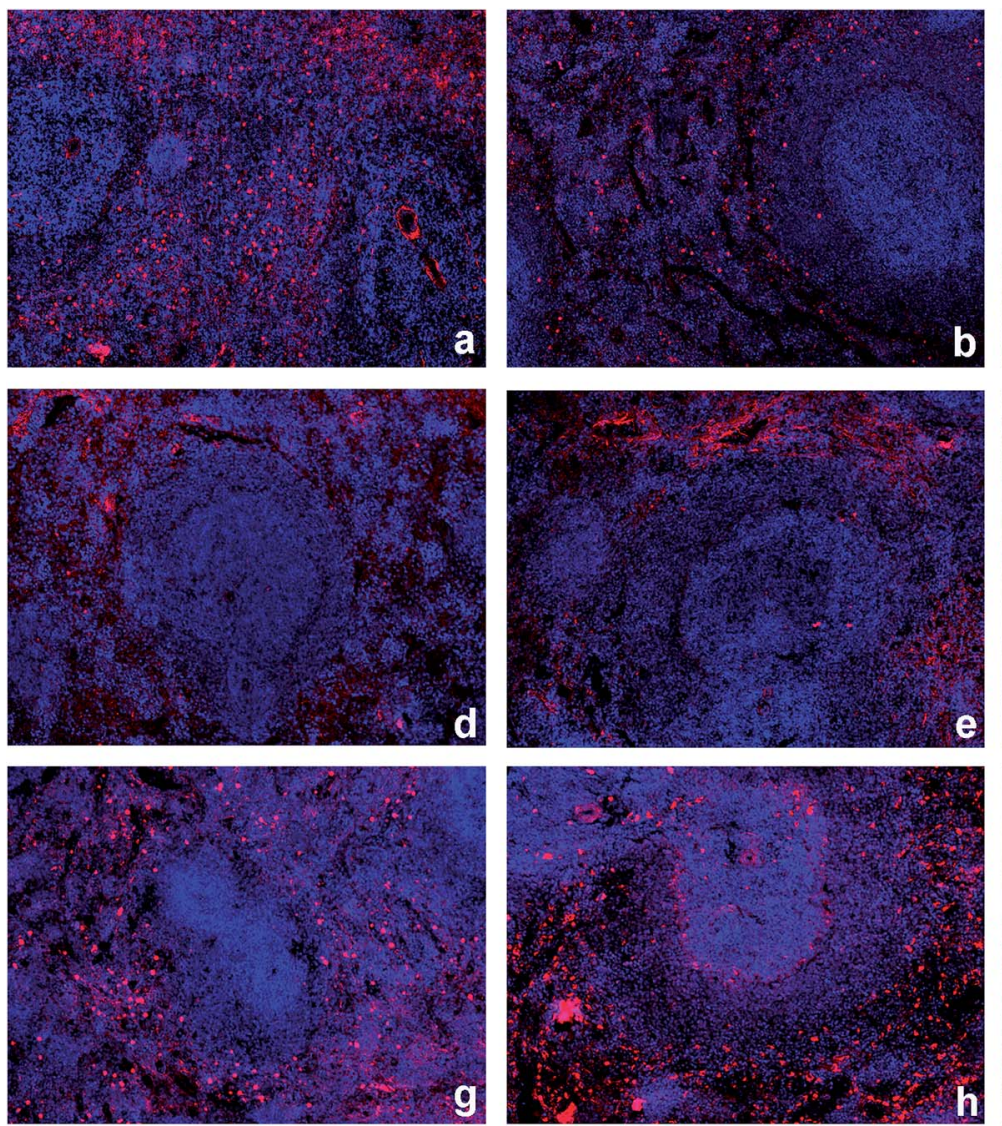
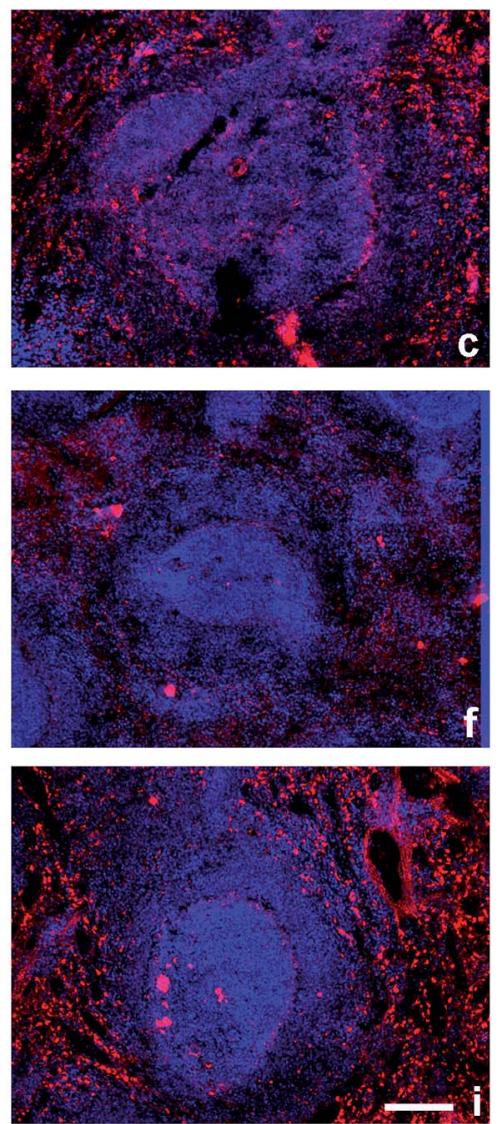

Fig. 4 Immunohistochemistry of splenic ferroportin. (a) Normal control. (b) IDA control. (c) IDA rats treated with FS. (d-f) IDA rats treated with Que, Qtg, Pat. (g-i) IDA rats treated with combination of QueFS, QtgFS, PatFS. Scale bar $50 \mu \mathrm{m}$.

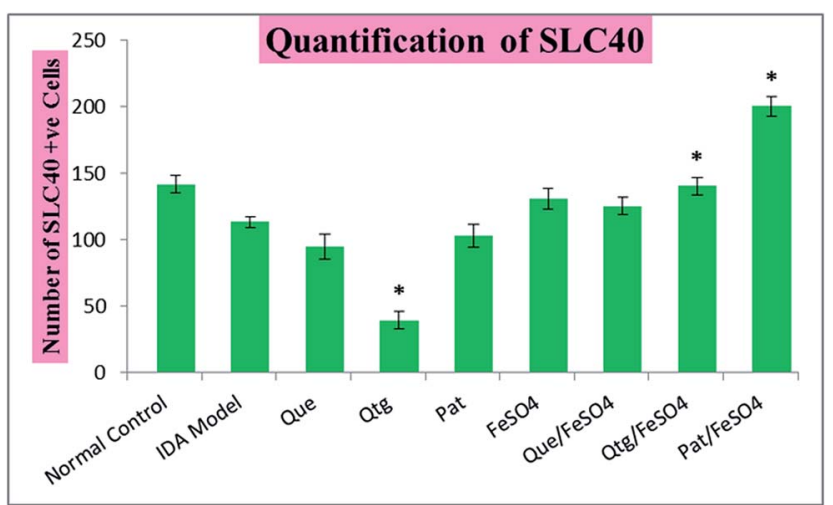

Fig. 5 Graphical representation of SLC-40 quantification in immunofluorescent photomicrographs of spleen. Iron deficiency anemia model group and standard $\left(\mathrm{FeSO}_{4}\right)$ group showed non-significant decrease and increase respectively in splenic ferroportin expression as compared to normal control group. Qtg significantly lowered the expression of ferroportin in IDA spleen. Whereas combination of QtgFS and PatFS showed significant rise in ferroportin expression as compared to IDA group. PatFS group is showing highest upregulation of ferroportin in spleen. Values are expressed as mean $\pm \operatorname{SEM}(n=10)$. Bars showing significant differences $(* p<0.05)$ compared to IDA model control. transcription level. ${ }^{34}$ Previously conducted in vitro as well as in vivo studies shown that acute exposure of quercetin suppresses the ferroportin expression in intestinal cells. ${ }^{20} \mathrm{We}$ also observed slightly lower ferroportin with QueFS combination than $\mathrm{FeSO}_{4}$ alone, however, QtgFS and PatFS increased the ferroportin. The reference data regarding effect of patuletin and quercetagetin in literature is lacking and this is the first time we observed their effects on ferroportin expression in our study. The underlying mechanism of flavonoid-mediated induction in ferroportin expression is not well understood. However, we may assume that polyphenols combined with iron supplementation possibly exert their effect on ferroportin expression through their antioxidant property and by inhibiting stimulated inflammatory mediators in the body such as TNF- $\alpha$, IL-1 $\beta, \mathrm{IL}-6, \mathrm{NF}-\kappa \mathrm{B}$, etc. ${ }^{35}$ Inflammatory stimuli inhibit expression of ferroportin such as through induction of hepcidin. ${ }^{36}$ Therefore, potential antioxidant ability of polyphenols combined the state of nutritional iron deficiency when body regulatory mechanisms to recover iron stores are activated, might allow the ferroportin expression to be increased.

\section{Conclusion}

In conclusion, concomitant use of flavonoids and $\mathrm{FeSO}_{4}$ improve hematological parameters, increase splenic tissue 
availability of iron and ferroportin expression in iron deficiency anemia; this effect seems to be variable with alterations in structural features of the flavonoids.

\section{Conflict of interest}

All authors have declared no conflicts of interest.

\section{References}

1 I. Alton, Iron deficiency anemia, Guide Lines for Adolescent Nutrition Services, Internet, 2005, pp. 101-108.

2 V. Cheynier, Polyphenols in foods are more complex than often thought, Am. J. Clin. Nutr., 2005, 81, 223S-229S.

3 P. A. Kroon, M. N. Clifford, A. Crozier, A. J. Day, J. L. Donovan, C. Manach and G. Williamson, How should we assess the effects of exposure to dietary polyphenols in vitro?, Am. J. Clin. Nutr., 2004, 80, 15-21.

4 K. M. Tuohy, L. Conterno, M. Gasperotti and R. Viola, Upregulating the human intestinal microbiome using whole plant foods, polyphenols, and/or fiber, J. Agric. Food Chem., 2012, 60, 8776-8782.

5 E. Gallagher, T. R. Gormley and E. K. Arendt, Recent advances in the formulation of gluten-free cereal-based products, Trends Food Sci. Technol., 2004, 15, 143-152.

6 Z. Tolkien, L. Stecher, A. P. Mander, D. I. Pereira and J. J. Powell, Ferrous sulfate supplementation causes significant gastrointestinal side-effects in adults: a systematic review and meta-analysis, PloS One, 2015, 10(2), e0117383.

7 T. Ganz and E. Nemeth, Iron imports. IV. Hepcidin and regulation of body iron metabolism, Am. J. Physiol.: Gastrointest. Liver Physiol., 2006, 290, G199-G203.

8 T. M. Steele, D. M. Frazer and G. J. Anderson, Systemic regulation of intestinal iron absorption, IUBMB Life, 2005, 57, 499-503.

9 A. M. Thomson, J. T. Rogers and P. J. Leedman, Iron-regulatory proteins, iron-responsive elements and ferritin mRNA translation, Int. J. Biochem. Cell Biol., 1999, 31, 1139-1152.

10 A. Donovan, C. A. Lima, J. L. Pinkus, G. S. Pinkus, L. I. Zon, S. Robine and N. C. Andrews, The iron exporter ferroportin/ Slc40a1 is essential for iron homeostasis, Cell Metab., 2005, 1, 191-200.

11 C. Beaumont, Multiple regulatory mechanisms act in concert to control ferroportin expression and heme iron recycling by macrophages, Haematologica, 2010, 95, 12331236.

12 D. L. Zhang, R. M. Hughes, H. Ollivierre-Wilson, M. C. Ghosh and T. A. Rouault, A ferroportin transcript that lacks an iron-responsive element enables duodenal and erythroid precursor cells to evade translational repression, Cell Metab., 2009, 9, 461-473.

13 C. Sangokoya, J. F. Doss and J. T. Chi, Iron-responsive miR485-3p regulates cellular iron homeostasis by targeting ferroportin, PLoS Genet., 2013, 9, e1003408, DOI: 10.1371/ journal.pgen.1003408.
14 A. Wach, K. Pyrzyńska and M. Biesaga, Quercetin content in some food and herbal samples, Food Chem., 2007, 100, 699-704.

15 P. A. Ruiz and D. Haller, Functional diversity of flavonoids in the inhibition of the proinflammatory NF- $\mathrm{B}$, IRF, and Akt signaling pathways in murine intestinal epithelial cells, $J$. Nutr., 2006, 136, 664-671.

16 M. V. Chaud, C. Izumi, Z. Nahaal, T. Shuhama, M. D. L. P. Bianchi and O. D. Freitas, Iron derivatives from casein hydrolysates as a potential source in the treatment of iron deficiency, J. Agric. Food Chem., 2002, 50, 871-877.

17 J. Zhou, X. Y. Mao, X. Wang, T. Ai, J. J. Ma and Y. H. Li, Antianaemia efficacy of $\beta$-lactoglobulin hydrolysate-iron complex on iron-deficient anaemic rats, Eur. J. Nutr., 2014, 53, 877-884.

18 A. A. Machado, C. Izumi, S. Zucoloto and O. Freitas, Iron depositions in rat liver and spleen were lower when the mineral was supplied as a derivative of casein hydrolysate in place of iron sulfate, Aliment. Nutr., 2009, 16, 111-116.

19 M. Symonowicz and M. Kolanek, Flavonoids and their properties to form chelate complexes, J. Microbiol., Biotechnol. Food Sci., 2012, 76, 35-41.

20 M. Lesjak, R. Hoque, S. Balesaria, V. Skinner, E. S. Debnam, S. K. Srai and P. A. Sharp, Quercetin inhibits intestinal iron absorption and ferroportin transporter expression in vivo and in vitro, PLoS One, 2014, 9, e102900, DOI: 10.1371/ journal.pone.0102900.

21 A. A. Jaccob, S. A. Hussain and S. A. Hussain, Effects of longterm use of flavonoids on the absorption and tissue distribution of orally administered doses of trace elements in rats, Pharmacol. Pharm., 2012, 3, 474-480, DOI: 10.4236/ pp.2012.34065.

22 M. M. Baccan, O. Chiarelli-Neto, R. M. S. Pereira and B. P. Espósito, Quercetin as a shuttle for labile iron, $J$. Inorg. Biochem., 2012, 107, 34-39.

23 Q. Ma, E. Y. Kim and O. Han, Bioactive dietary polyphenols decrease heme iron absorption by decreasing basolateral iron release in human intestinal Caco-2 cells, J. Nutr., 2010, 140, 1117-1121.

24 C. F. Skibola and M. T. Smith, Potential health impacts of excessive flavonoid intake, Free Radical Biol. Med., 2000, 29, 375-383.

25 P. C. Hollman, J. M. van Trijp, M. N. Buysman, M. J. Mengelers, J. H. de Vries and M. B. Katan, Relative bioavailability of the antioxidant flavonoid quercetin from various foods in man, FEBS Lett., 1997, 418, 152-156.

26 C. Coudray, J. C. Tressol, C. Feillet-Coudray, J. Bellanger, D. Pépin and A. Mazur, Long-term consumption of red wine does not modify intestinal absorption or status of zinc and copper in rats, J. Nutr., 2000, 130, 1309-1313.

27 G. J. Lee, I. A. Cho, K. R. Kang, D. K. Kim, H. M. Sohn, J. W. You, J. S. Oh, Y. S. Seo, S. J. Yu, J. S. You and C. S. Kim, Biological Effects of the Herbal Plant-Derived Phytoestrogen Bavachin in Primary Rat Chondrocytes, Biol. Pharm. Bull., 2014, 38, 1199-1207.

28 S. Upadhyay and M. Dixit, Role of polyphenols and other phytochemicals on molecular signaling, Oxid. Med. Cell. Longevity, 2015, 504253, DOI: 10.1155/2015/504253. 
29 R. Henneberg, M. F. Otuki, A. E. F. Furman, P. Hermann, A. J. D. Nascimento and M. S. S. Leonart, Protective effect of flavonoids against reactive oxygen species production in sickle cell anemia patients treated with hydroxyurea, Rev. Bras. Hematol. Hemoter., 2013, 35, 52-55.

30 E. Vlachodimitropoulou, P. A. Sharp and R. J. Naftalin, Quercetin-iron chelates are transported via glucose transporters, Free Radical Biol. Med., 2011, 50, 934-944.

31 D. G. Salinas, M. De La Fuente and J. G. Reyes, Changes of enzyme activity in lipid signaling pathways related to substrate reordering, Biophys. J., 2005, 89, 885-894.

32 M. C. D'Anna, T. V. Veuthey and M. E. Roque, Immunolocalization of ferroportin in healthy and anemic mice, J. Histochem. Cytochem., 2009, 57, 9-16.
33 E. Gammella, S. Recalcati and G. Cairo, Dual Role of ROS as Signal and Stress Agents: Iron Tips the Balance in Favor of Toxic Effects, Oxid. Med. Cell. Longevity, 2016, 8629024, DOI: $10.1155 / 2016 / 8629024$.

34 F. Aydemir, S. Jenkitkasemwong, S. Gulec and M. D. Knutson, Iron loading increases ferroportin heterogeneous nuclear RNA and mRNA levels in murine J774 macrophages, J. Nutr., 2009, 139, 434-438.

35 S. Patel and M. Vajdy, Induction of cellular and molecular immunomodulatory pathways by vitamin A and flavonoids, Expert Opin. Biol. Ther., 2015, 15, 1411-1428.

36 J. F. Collins, M. Wessling-Resnick and M. D. Knutson, Hepcidin regulation of iron transport, J. Nutr., 2008, 138, 2284-2288. 